CONFORMAL GEOMETRY AND DYNAMICS

An Electronic Journal of the American Mathematical Society

Volume 3, Pages 67-78 (June 14,1999)

S $1088-4173(99) 00045-4$

\title{
FAMILIES OF BAKER DOMAINS II
}

\author{
P. J. RIPPON AND G. M. STALLARD
}

\begin{abstract}
Let $f$ be a transcendental meromorphic function and $U$ be an invariant Baker domain of $f$. We use estimates for the hyperbolic metric to show that there is a relationship between the size of $U$ and the proximity of $f$ in $U$ to the identity function, and illustrate this by discussing how the dynamics of transcendental entire functions of the following form vary with the parameter $a$ :

$f(z)=a z+b z^{k} e^{-z}(1+o(1))$ as $\Re(z) \rightarrow \infty$,

where $k \in \mathbf{N}, a \geq 1$ and $b>0$.
\end{abstract}

\section{INTRODUCTION}

Let $f$ be a meromorphic function which is not rational of degree one and denote by $f^{n}, n \in \mathbf{N}$, the $n$th iterate of $f$. The set of normality, $N(f)$, is defined to be the set of points, $z \in \mathbf{C}$, such that $\left(f^{n}\right)_{n \in \mathbf{N}}$ is well-defined, meromorphic and forms a normal family in some neighbourhood of $z$. The complement, $J(f)$, of $N(f)$ is called the Julia set of $f$. An introduction to the properties of these sets can be found in, for example, [3] for rational functions and in [4] for transcendental meromorphic functions.

The set $N(f)$ is completely invariant so that, if $U$ is a component of $N(f)$, then, for each $p \in \mathbf{N}$, there exists a component $U_{p}$ of $N(f)$ such that $f^{p}(U) \subset U_{p}$. If $U_{p} \neq U_{m}$, for each $p \neq m$, then we say that $U$ is a wandering domain. If $U_{p}=U$, then we say that $U$ is a periodic component of period $p$ (assuming $p$ to be minimal) and there are then five possibilities (see, for example, [4]). In particular, $U$ is called a Baker domain or an essentially parabolic domain if there exists $z_{0} \in \partial U$ such that $f^{n p}(z) \rightarrow z_{0}$ as $n \rightarrow \infty$, for $z \in U$, but $f^{p}\left(z_{0}\right)$ is not defined.

If $U$ is a Baker domain, then $f$ must be transcendental. If $f$ is in fact a transcendental entire function, then $f^{n p}(z) \rightarrow \infty$ as $n \rightarrow \infty$ for $z \in U$ and, moreover, $U$ is simply connected [1, Theorem 3.1]. This is not true in general for transcendental meromorphic functions - for example, in [8] it is shown that the function $f(z)=z+2+e^{-z}+(100(z-(1+i \pi)))^{-1}$ has a multiply connected Baker domain.

Information about the rate at which iterates tend to infinity in a Baker domain can be obtained by using estimates for the hyperbolic metric. For example, it was shown by Baker (see, for example, [4, Lemma 7]) that, if $U$ is a simply connected invariant Baker domain, and $z_{0} \in U$, then for any path $\Gamma=\bigcup_{n=0}^{\infty} f^{n}\left(\Gamma_{0}\right)$, where $\Gamma_{0}$ joins $z_{0}$ to $f\left(z_{0}\right)$ in $U$ and $0 \notin \Gamma$, there is a constant $C$ such that

$$
|f(z)| \leq C|z|, \text { for } z \in \Gamma \text {. }
$$

Received by the editors January 5, 1999 and, in revised form, April 19, 1999.

1991 Mathematics Subject Classification. Primary 30D05; Secondary 58F08.

(C)1999 American Mathematical Society 
In particular, $\left|f^{n}\left(z_{0}\right)\right| \leq C^{n}\left|z_{0}\right|$, for each $n \in \mathbf{N}$.

Various examples of functions $f$ with Baker domains $U$ are known (see, for example, [2], [5], [9], [10], [11], [13] and [15]) and these examples suggest a connection between the proximity of $f$ in $U$ to the identity function and the thinness of $U$ itself. For example, (see [9] and [15]) the function $f(z)=z+e^{-z}$ has a Baker domain $U_{m}$ in each strip

$$
\{z:|\Im(z)-2 m \pi|<\pi\}, m \in \mathbf{Z},
$$

and $U_{m}$ has asymptotic width $2 \pi$ as $\Re(z) \rightarrow \infty$, whereas the function $f(z)=$ $z+\exp \left(-e^{z}\right)$ has an infinite family of (much thinner) Baker domains in each such strip.

In Section 2 of this paper, we use standard estimates for the hyperbolic metric to obtain the following result which confirms such a connection. We use the following notation throughout the paper:

- $B(z, r)=\{w:|w-z|<r\}$

- $d_{U}(z)=\inf \{|z-w|: w \in \partial U\}$

- $[z, w]_{D}$ is the distance from $z$ to $w$ with respect to the hyperbolic metric in the domain $D$;

- $z_{n}=f^{n}\left(z_{0}\right)$, where $z_{0}$ and $f$ are given.

Theorem 1. Let $f$ be a transcendental meromorphic function, $U$ be an invariant Baker domain of $f$ and $z_{0} \in U$.

(a) If $\left[z_{n+1}, z_{n}\right]_{U} \nrightarrow 0$, then there exists $C>0$ such that

$$
B\left(z_{n}, C\left|z_{n+1}-z_{n}\right|\right) \cap U^{c} \neq \emptyset, \text { for } n \geq 0 .
$$

(b) If $U$ is simply connected, and $\Gamma=\bigcup_{n=0}^{\infty} f^{n}\left(\Gamma_{0}\right)$, where $\Gamma_{0}$ is a path in $U$ joining $z_{0}$ to $z_{1}$, then there exists $c>0$ such that

$$
U \supset \bigcup_{z \in \Gamma} B(z, c|f(z)-z|) .
$$

Remarks. 1. Later (Theorem 3) we use Theorem 1 part (b) to show the nonexistence of a Baker domain under particular circumstances.

2. The sequence $\left[z_{n+1}, z_{n}\right]_{U}$ in part (a) is decreasing since, for $z, w \in U$,

$$
[f(z), f(w)]_{U} \leq[f(z), f(w)]_{f(U)} \leq[z, w]_{U},
$$

by $\left[7\right.$, Theorem 4.1]. Whether or not $\left[z_{n+1}, z_{n}\right]_{U} \rightarrow 0$ is independent of the choice of $z_{0} \in U$ if $U$ is simply connected. To see why this is true, we take $U$ to be the right half-plane $H$, without loss of generality. In [14] it was shown that, if $\left[z_{n+1}, z_{n}\right]_{H} \nrightarrow 0$, for some $z_{0} \in H$, then there exists a function $g$ that is analytic in $H$ with $\Re(g)>0$ in $H$ such that

$$
g(f(z))=g(z)+i, \text { for } z \in H .
$$

Thus $g\left(z_{n+1}\right)=g\left(z_{n}\right)+i$, for each $n \in \mathbf{N}$ and any $z_{0} \in H$ so that

$$
\left[z_{n+1}, z_{n}\right]_{H} \geq\left[g\left(z_{n+1}\right), g\left(z_{n}\right)\right]_{g(H)} \geq\left[g\left(z_{n+1}\right), g\left(z_{n}\right)\right]_{H} \nrightarrow 0 .
$$

The example $f(z)=z+e^{-z}$ mentioned earlier shows that some condition such as $\left[z_{n+1}, z_{n}\right]_{U} \nrightarrow 0$ is needed to obtain the conclusion in part (a) of Theorem 1 . The condition $\left[z_{n+1}, z_{n}\right]_{U} \not 0$ is certainly satisfied if $U$ is simply connected and $f$ is univalent in $U$. This suggests the question of whether there exists a function $f$ with Baker domain $U$ such that $\left[z_{n+1}, z_{n}\right]_{U} \nrightarrow 0$ but $f$ is not univalent in $U$. It is 
straightforward to check that, if $f(z)=2 z+e^{-z}$, then $f$ has a simply connected invariant Baker domain $U$ containing $\{z: \Re(z)>1\} \cup\{z: \Im(z)=0\}$. Similar arguments to those used in the proof of Theorem 2 part (b) below show that $\left[z_{n+1}, z_{n}\right]_{U} \nrightarrow 0$, if $z_{0} \in U$. The set of critical points of $f$ is

$$
\{z: z=-\ln 2+2 m \pi i \text {, for some } m \in \mathbf{Z}\}
$$

and so $f$ is certainly not univalent in $U$, as $-\ln 2 \in U$. In fact, since $f(z)=$ $2 z+\phi(z)$, where $\phi(z+2 \pi i)=\phi(z)$, it follows from the main result of [6] (see also [16, Corollary 1]) that $N(f)$ is invariant under translation by $2 \pi i$ and so $U$ contains all of the critical points of $f$ and, indeed, all of the infinitely many critical values of $f$.

There is interest (see, for example, [4]) in establishing the relationship between the Baker domains of a function $f$ and the set of singularities of $f^{-1}$, which consists of the critical values and finite asymptotic values of $f$. In Section 3, we use what seems to be a new technique to show that entire functions in a certain large class have Baker domains which contain infinitely many such singularities, and in which the hyperbolic distance between successive iterates of points does not tend to zero.

Theorem 2. Let $f$ be a transcendental entire function of the form

$$
f(z)=a z+b z^{k} e^{-z}(1+o(1)) \text { as } \Re(z) \rightarrow \infty,
$$

where $k \in \mathbf{N}, a>1$ and $b>0$. Then

(a) $f$ has a simply connected invariant Baker domain $U$ which, for each $\rho>0$ and large values of $R>0$, contains an invariant set of the form

$$
D_{\rho, R}=\left\{z:\left|z^{k} e^{-z}\right|<\rho,|z|>R\right\}
$$

(b) $\left[z_{n+1}, z_{n}\right]_{U} \nrightarrow 0$ as $n \rightarrow \infty$, for each $z_{0} \in U$;

(c) $f$ is not univalent in $U$ and, moreover, there are infinitely many singularities of $f^{-1}$ in $U$, each of which corresponds to a critical point or an asymptotic path of $f$ in $U$.

Remarks. 1. If $f$ is of the form (1.2) with $k \in \mathbf{Z} \backslash \mathbf{N}$, then it is easy to check that $f$ has an invariant Baker domain $U$ which, for large values of $R$, contains an invariant set of the form $\{z: \Re(z)>R\}$. Part (c) of Theorem 2 is, however, not true in general for such $k$. For example, $f(z)=2 z+2 e^{-2} e^{-z}$ has such an invariant Baker domain $U$ and $f$ is univalent in $U$. This follows from the corresponding properties of $f(z)=2 z+2-\ln 2-e^{z}[5$, Theorems 1 and 2$]$ by making the change of variable $w=-z+\ln 2-2$.

2. In [15], we showed that if $f$ is of the form (1.2) with $a=1$, then for each $m \in \mathbf{Z}$, there is an invariant Baker domain $U_{m}$ of $f$ such that, for each $0<\theta<\pi$, $U_{m}$ contains a set of the form

$$
V_{m}(\theta)=\left\{x+i y: x>v_{m}(\theta),|y-2 m \pi|<\theta\right\} .
$$

For $z_{0} \in U_{m}, \Re\left(z_{n}\right) \rightarrow \infty$ and $\Im\left(z_{n}\right) \rightarrow 2 m \pi$, so that $\left[z_{n+1}, z_{n}\right]_{U_{m}} \rightarrow 0$ as $n \rightarrow \infty$, the $U_{m}$ are distinct, and each contains a singularity of $f^{-1}$. Thus the change in the dynamics of functions of the form (1.2) as a decreases to 1 is analogous to the change in dynamical behaviour occurring at a parabolic bifurcation, in that a single basin of attraction at infinity is replaced by infinitely many such basins in which convergence to infinity is much slower. 
The following diagrams illustrate this change for $f(z)=a z\left(1+e^{-z}\right)$ with $a=1.01$ and $a=1$. In both cases, points of $\{x+i y:|x| \leq 12,|y| \leq 12\}$ have been plotted red or yellow if their forward iterates under $f$ become large very quickly, suggesting that they do not lie in an invariant domain for $f$, and black otherwise. Evidence for the location of Baker domains is provided by the forward orbits of many points on $x=4$, which are plotted in white.

Figures 1 and 2 were produced using the software $\mathrm{C}++$ Builder, and the authors are grateful to Bob Margolis and Toni Cokayne (Department of Pure Mathematics, Open University) for help with this.

In Section 4, we use Theorem 2 to analyse the dynamics of a particular family of examples. Note that the proof of uniqueness in Theorem 3 part (b) below uses Theorem 1 part (b).

Theorem 3. Let $f(z)=a z\left(1+e^{-z^{p}}\right)$, where $a>1$ and $p \in \mathbf{N}$. Then, for $k \in$ $\{0,1, \ldots, p-1\}$,

(a) $\{z: \arg z=(2 k+1) \pi / p\} \subset J(f)$;

(b) $f$ has a unique simply connected invariant Baker domain $U_{k}$ in

$$
A_{k}=\left\{z:|\arg z-2 k \pi / p|<\frac{\pi}{p}\right\}
$$

which, for each $\epsilon, 0<\epsilon<\frac{\pi}{2 p}$, contains a set of the form

$$
\left\{z:|\arg z-2 k \pi / p|<\frac{\pi}{2 p}-\epsilon,|z|>R\right\}
$$

(c) $U_{k}$ contains infinitely many critical points of $f$.

In [15], we showed that the function $g(z)=e^{2 \pi i / p} z\left(1+e^{-z^{p}}\right), p \in \mathbf{N}$, has infinitely many $p$-cycles of rather thin Baker domains. These were the first examples of entire functions having Baker domains which are not invariant. The following corollary to Theorem 3 shows that a small change to this function gives an entire function with a $p$-cycle of Baker domains which are comparable in size to a sector.

Corollary 1. For $p \in \mathbf{N}$ and $a>1$, let $g(z)=a e^{2 \pi i / p} z\left(1+e^{-z^{p}}\right)$. Then the sets $U_{k}, k=0,1, \ldots, p-1$, in Theorem 3 form a $p$-cycle of Baker domains for $g$, and each $U_{k}$ contains infinitely many critical points of $g$.

Indeed, $g^{n}(z)=\omega^{n} f^{n}(z)$, for $n \in \mathbf{N}$, where $\omega=e^{2 \pi i / p}$ and $f$ is the function of Theorem 3. In particular, $g^{p}=f^{p}$ so that $J(g)=J(f)$, and it follows that the Baker domains of $g$ and of $f$ must coincide.

The functions in Corollary 1 are not univalent on their Baker domains. We end, in Section 5, by showing how an approximation theory method used by Eremenko and Lyubich in [10] can be adapted to prove the following result.

Theorem 4. For each $p \in \mathbf{N}$, there exists an entire function $f$ which has a $p$-cycle of Baker domains on which $f$ is univalent.

The proof of Theorem 4 yields Baker domains of a similar size (namely, comparable to a sector) to those in Theorem 3 and Corollary 1. 


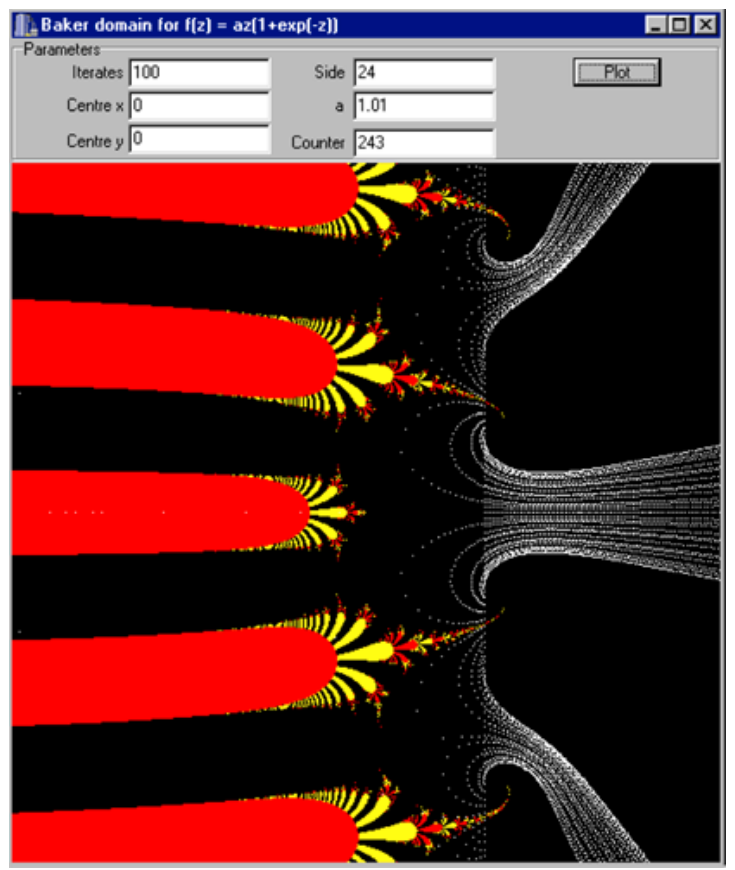

FiguRE 1. $a=1.01$

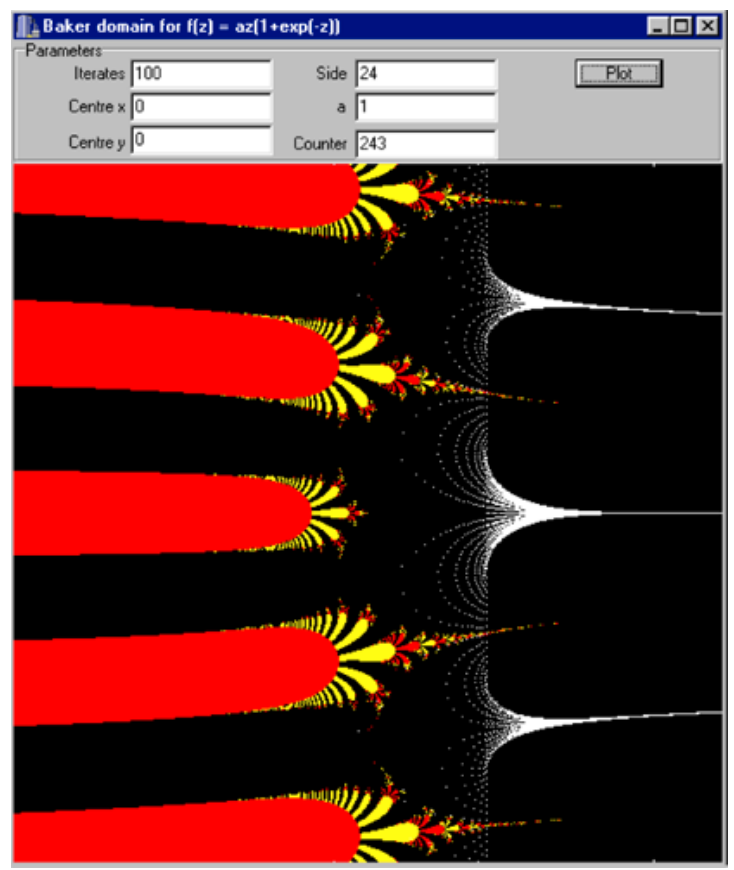

FiguRE 2. $a=1$ 


\section{Proof of Theorem 1}

We deduce Theorem 1 from the following standard estimates for hyperbolic distance.

Lemma 2.1. (a) If $z, w$ belong to a domain $U$, then

$$
|w-z| \geq d_{U}(z)\left(\frac{2}{[w, z]_{U}}+1\right)^{-1} .
$$

(b) If, furthermore, $U$ is simply connected, then

$$
|w-z| \leq d_{U}(z)\left(\exp \left(2[w, z]_{U}\right)-1\right) .
$$

Proof. We begin by supposing that

$$
d_{U}(z)>\left(\frac{2}{[w, z]_{U}}+1\right)|w-z| \text {. }
$$

If $L$ is the line segment from $z$ to $w$, then $L \subset U$ and, for $\xi \in L$,

$$
d_{U}(\xi) \geq d_{U}(z)-|\xi-z|>\frac{2}{[w, z]_{U}}|w-z|
$$

so that, by [7, Theorem 4.3], for example,

$$
[w, z]_{U} \leq \int_{L} \rho_{U}(\xi)|d \xi| \leq 2 \int_{L} \frac{|d \xi|}{d_{U}(\xi)}<\frac{2|w-z|}{2|w-z|}[w, z]_{U},
$$

where $\rho_{U}$ denotes the density of the hyperbolic metric in $U$. This contradiction proves part (a).

Now suppose that $U$ is simply connected and $\gamma$ is a hyperbolic geodesic in $U$ from $z$ to $w$. Then, by [7, Theorem 4.3], for example,

$$
[w, z]_{U}=\int_{\gamma} \rho_{U}(\xi)|d \xi| \geq \frac{1}{2} \int_{\gamma} \frac{|d \xi|}{d_{U}(\xi)} \geq \frac{1}{2} \int_{\gamma} \frac{|d \xi|}{d_{U}(z)+|z-\xi|}
$$

Now

$$
\gamma \cap\{\xi:|\xi-z|=t\} \neq \emptyset, \text { for } 0 \leq t \leq|w-z|
$$

and so

$$
[w, z]_{U} \geq \frac{1}{2} \int_{0}^{|w-z|} \frac{d t}{d_{U}(z)+t}=\frac{1}{2} \log \left(1+\frac{|w-z|}{d_{U}(z)}\right)
$$

as required.

We now show how Theorem 1 follows from Lemma 2.1. From part (a) of Lemma 2.1 ,

$$
d_{U}\left(z_{n}\right) \leq\left|z_{n+1}-z_{n}\right|\left(\frac{2}{\left[z_{n+1}, z_{n}\right]_{U}}+1\right) .
$$

So, if $\left[z_{n+1}, z_{n}\right]_{U} \nrightarrow 0$, then there exists $C>0$ such that

$$
d_{U}\left(z_{n}\right)<C\left|z_{n+1}-z_{n}\right|
$$

for $n \geq 0$. This proves part (a) of Theorem 1 .

If $z \in \Gamma$, then $z=f^{n}\left(\xi_{0}\right)$, for some $\xi_{0} \in \Gamma_{0}, n \geq 0$. Now

$$
[f(z), z]_{U}=\left[f^{n+1}\left(\xi_{0}\right), f^{n}\left(\xi_{0}\right)\right]_{U} \leq\left[f\left(\xi_{0}\right), \xi_{0}\right]_{U} \leq \sup _{\xi \in \Gamma_{0}}[f(\xi), \xi]_{U}=d,
$$

say, and so part (b) of Theorem 1 follows from part (b) of Lemma 2.1, on taking $c=\left(e^{2 d}-1\right)^{-1}$. 


\section{Proof of Theorem 2}

Let $f$ be a transcendental entire function of the form

$$
f(z)=a z+b z^{k} e^{-z}(1+o(1)) \text { as } \Re(z) \rightarrow \infty,
$$

where $a>1, b>0$ and $k \in \mathbf{N}$. We begin our proof of Theorem 2 with the following key result.

Lemma 3.1. Let

$$
D_{\rho, R}=\left\{z:\left|z^{k} e^{-z}\right|<\rho,|z|>R\right\} .
$$

For each $\rho>0$, there exists $R(\rho)>0$ such that

$$
f\left(D_{\rho, R}\right) \subset D_{\rho, R},
$$

for each $R>R(\rho)$.

Proof. First note that

$$
\left|z^{k} e^{-z}\right|<\rho \Longleftrightarrow \Re(z)>k \ln |z|-\ln \rho
$$

so that, for any fixed $\rho>0$,

$$
\min \left\{\Re(z): z \in D_{\rho, R}\right\} \rightarrow \infty \text { as } R \rightarrow \infty .
$$

Thus, if $\rho>0$ is fixed, then $R$ can be chosen so large that, for $z \in D_{\rho, R}$,

$$
|f(z)| \geq a|z|-2 b\left|z^{k} e^{-z}\right| \geq \frac{1}{2}(a+1)|z|>R
$$

and

$$
|f(z)| \leq a|z|+2 b\left|z^{k} e^{-z}\right| \leq 2 a|z|
$$

Now, by (3.1) and (3.2), if $z=x+i y \in D_{\rho, R}$, then $f(z)=X+i Y \in D_{\rho, R}$ if and only if

$$
X>k \ln |f(z)|-\ln \rho .
$$

It follows from (3.1) and (3.3) that, if $R$ is sufficiently large, then this is true for any $z \in D_{\rho, R}$, since

$$
\begin{aligned}
X & \geq a x-2 b\left|z^{k} e^{-z}\right| \\
& >a x-2 b \rho \\
& >a(k \ln |z|-\ln \rho)-2 b \rho \\
& =k \ln |z|+(a-1) k \ln |z|-a \ln \rho-2 b \rho \\
& >k \ln (2 a|z|)-\ln \rho \\
& >k \ln |f(z)|-\ln \rho .
\end{aligned}
$$

This proves Lemma 3.1.

Theorem 2 part (a) follows from Lemma 3.1, Montel's Theorem and the fact that $f$ is entire.

To prove part (b), we fix $\rho>0$ and take $z_{0} \in D_{\rho, R}$. If $R$ is sufficiently large, then $z_{0} \in U$ and it follows from Lemma 3.1 and (3.2) that, for each $n \in \mathbf{N}$,

$$
\left|z_{n+1}\right| \geq \frac{1}{2}(a+1)\left|z_{n}\right|
$$

Fixing a point $w \in J(f)$, we note that, since $\left|z_{n}\right| \rightarrow \infty$ as $n \rightarrow \infty$,

$$
d_{U}\left(z_{n}\right) \leq\left|z_{n}-w\right| \leq 2\left|z_{n}\right|
$$


for large values of $n$. Since $U$ is simply connected, it follows from Lemma 2.1 part (b) and the above inequalities that, for large $n$,

$$
\exp \left(2\left[z_{n+1}, z_{n}\right]_{U}\right) \geq \frac{\left|z_{n+1}-z_{n}\right|}{d_{U}\left(z_{n}\right)}+1 \geq \frac{a-1}{4}+1
$$

and so $\left[z_{n+1}, z_{n}\right]_{U} \nrightarrow 0$ as $n \rightarrow \infty$. It now follows from the second remark after Theorem 1 that, for any $z_{0} \in U,\left[z_{n+1}, z_{n}\right]_{U} \not \rightarrow 0$, as required.

The proof of part (c) of Theorem 2 uses the following result.

Lemma 3.2. Let $\rho>6 a \pi / b$. If $R$ is sufficiently large, then for each $n \in \mathbf{N}$, there exists a subarc $\gamma_{n}$ of $\partial D_{\rho, R}$ such that $f\left(\gamma_{n}\right)$ is a closed curve and $\min \{\Im(f(z))$ : $\left.z \in \gamma_{n}\right\} \rightarrow \infty$ as $n \rightarrow \infty$.

Proof. Let $C_{\rho, R}$ denote that part of $\partial D_{\rho, R}$ which lies in the upper half-plane but not on $\{z:|z|=R\}$. If $z=x+i y \in C_{\rho, R}$, then

$$
x=k \ln |z|-\ln \rho,
$$

and

$$
\theta(z)=k \arg z-y
$$

is a continuous argument of $z^{k} e^{-z}$ on $C_{\rho, R}$. Here $\arg z$ denotes the principal argument, and we note that $\arg z \rightarrow \pi / 2$ as $z \rightarrow \infty$ along $C_{\rho, R}$. Thus, for $n \geq n_{0}$ say, there exist $\xi_{n}, \xi_{n}^{\prime}, \xi_{n}^{\prime \prime}$ on $C_{\rho, R}$ with

$$
\theta\left(\xi_{n}\right)=-2 \pi n, \quad \theta\left(\xi_{n}^{\prime}\right)=-2 \pi n+3 \pi / 2, \quad \theta\left(\xi_{n}^{\prime \prime}\right)=-2 \pi n-3 \pi / 2 .
$$

For $n \geq n_{0}$, we put $\Gamma_{n}=\Gamma_{n}^{\prime} \cup \Gamma_{n}^{\prime \prime}$, where

$$
\Gamma_{n}^{\prime}=\left\{z \in C_{\rho, R}: 0 \leq \theta(z)+2 \pi n \leq 3 \pi / 2\right\}
$$

and

$$
\Gamma_{n}^{\prime \prime}=\left\{z \in C_{\rho, R}:-3 \pi / 2 \leq \theta(z)+2 \pi n \leq 0\right\} .
$$

We also put

$$
f_{n}(z)=f(z)-a \xi_{n}=a\left(z-\xi_{n}\right)+b z^{k} e^{-z}+b z^{k} e^{-z} \epsilon_{n}(z) .
$$

By the hypotheses of Theorem 2, we may assume that $n_{0}$ is so large that

$$
\left|z-\xi_{n}\right|<2 \pi \text { and }\left|b z^{k} e^{-z} \epsilon_{n}(z)\right|<\pi a / 4, \text { for } z \in \Gamma_{n}, n \geq n_{0} .
$$

Since $\rho>6 a \pi / b$, it follows from (3.5) that, for $z \in \Gamma_{n}, n \geq n_{0}$,

$$
\left|f_{n}(z)\right|>b \rho-3 \pi a>b \rho / 2,
$$

and with an appropriate continuous choice of $\arg \left(f_{n}(z)\right)$,

$$
\left|\arg \left(f_{n}(z)\right)-(\theta(z)+2 \pi n)\right|<\tan ^{-1}\left(\frac{3 \pi a}{b \rho}\right)<\tan ^{-1}(1 / 2)<\pi / 6 .
$$

We note that

$$
\Im\left(z-\xi_{n}\right)\left\{\begin{array}{l}
\leq 0, \quad \text { for } z \in \Gamma_{n}^{\prime} \\
\geq 0, \quad \text { for } z \in \Gamma_{n}^{\prime \prime}
\end{array}\right.
$$


and so it follows from (3.5) that

$$
\Im\left(f_{n}(z)\right) \begin{cases}<b \rho+\pi a / 4, & \text { for } z \in \Gamma_{n}^{\prime}, \\ >-b \rho-\pi a / 4, & \text { for } z \in \Gamma_{n}^{\prime \prime} .\end{cases}
$$

We may also assume that, for $n \geq n_{0}$,

$$
\Im\left(\xi_{n}-\xi_{n}^{\prime}\right) \geq \pi / 2 \text { and } \Im\left(\xi_{n}^{\prime \prime}-\xi_{n}\right) \geq \pi / 2,
$$

so that by (3.4) and (3.5),

$$
\Im\left(f_{n}\left(\xi_{n}^{\prime}\right)\right)<-b \rho-\pi a / 4 \text { and } \Im\left(f_{n}\left(\xi_{n}^{\prime \prime}\right)\right)>b \rho+\pi a / 4 .
$$

Now consider the maximal subarc $l_{n}$ of $f_{n}\left(\Gamma_{n}\right)$ which contains $f_{n}\left(\xi_{n}\right)=b \rho\left(1+\epsilon_{n}\left(\xi_{n}\right)\right)$ and lies in the strip $\{w:|\Im(w)| \leq b \rho+\pi a / 4\}$. In view of (3.7), (3.8) and (3.9), $l_{n}$ has endpoints $w_{n}^{\prime}=f_{n}\left(\eta_{n}^{\prime}\right)$ and $w_{n}^{\prime \prime}=f_{n}\left(\eta_{n}^{\prime \prime}\right)$, where

$$
\Im\left(w_{n}^{\prime}\right)=-b \rho-\pi a / 4, \quad \Im\left(w_{n}^{\prime \prime}\right)=b \rho+\pi a / 4 .
$$

Thus

$$
\arg \left(w_{n}^{\prime}\right)>\pi \text { and } \arg \left(w_{n}^{\prime \prime}\right)<-\pi
$$

so that

$$
5 \pi / 6<\theta\left(\eta_{n}^{\prime}\right)+2 \pi n<3 \pi / 2 \text { and }-3 \pi / 2<\theta\left(\eta_{n}^{\prime \prime}\right)+2 \pi n<-5 \pi / 6 .
$$

Then let $L_{n}$ denote the closed curve consisting of $l_{n}$ together with a segment each from the lines

$$
\{w: \Im(w)=-b \rho-\pi a / 4\}, \quad\{w: \Im(w)=b \rho+\pi a / 4\}, \quad\left\{w: \Re(w)=\mu_{n}\right\},
$$

where $\Re(f(z))<\mu_{n}$, for $z \in \Gamma_{n}$. By (3.6) and (3.7), $L_{n}$ winds exactly twice round $\{w:|w| \leq b \rho / 2\}$, and so is not simple. Thus $l_{n}$ is not simple and so $\Gamma_{n}$ must have a subarc $\gamma_{n}$ such that $f_{n}\left(\gamma_{n}\right)$ is closed and lies in the strip $\{w:|\Im(w)| \leq b \rho+\pi a / 4\}$. This is sufficient to prove Lemma 3.2.

Lemma 3.2 shows that $f$ is not univalent in $U$. To complete the proof of Theorem 2 part (c), we now take a sequence of arcs $\gamma_{n}$ satisfying the conditions of Lemma 3.2 together with an $\epsilon$-neighbourhood $G_{n}$ of each arc $\gamma_{n}$ such that $G_{n} \subset U$. Since $U$ is simply connected, the union $\Omega_{n}$ of $f\left(G_{n}\right)$ with the bounded complementary components of $f\left(G_{n}\right)$ is a bounded, simply connected subset of $U$. We claim that $\Omega_{n}$ contains a singularity of $f^{-1}$ corresponding to a critical point or asymptotic path of $f$ in $U$. Otherwise, the branch of $f^{-1}$ mapping $f\left(\alpha_{n}\right)$ to $\alpha_{n}$, where $\alpha_{n}$ is an endpoint of $\gamma_{n}$, can be continued along all paths in $\Omega_{n}$ to give a single-valued analytic function in $\Omega_{n}$ with values in $U$, and this is impossible since $f\left(\gamma_{n}\right) \subset \Omega_{n}$. This completes the proof of Theorem 2 .

\section{Proof of Theorem 3}

Let $f(z)=a z\left(1+e^{-z^{p}}\right)$, where $a>1$ and $p \in \mathbf{N}$. To prove part (a) of Theorem 3 , note that, if $z=t e^{i(2 k+1) \pi / p}$, where $t>0$ and $k \in\{0,1, \cdots, p-1\}$, then

$$
f(z)=a t e^{i(2 k+1) \pi / p}\left(1+e^{t^{p}}\right) .
$$

Thus

$$
f^{n}(z)=\phi^{n}(t) e^{i(2 k+1) \pi / p}
$$


where

$$
\phi(t)=a t\left(1+e^{t^{p}}\right)
$$

A simple calculation shows that $\phi^{\prime}(t) \geq \frac{2 \phi(t) \ln \phi(t)}{t \ln t}$, for large values of $t$, and it follows by integration (see [16]) that, if $t_{1}$ is sufficiently large and $t_{2}>t_{1}$, then

$$
\frac{\ln \phi^{n}\left(t_{2}\right)}{\ln \phi^{n}\left(t_{1}\right)} \rightarrow \infty \quad \text { as } n \rightarrow \infty
$$

Thus, if $z_{1}=t_{1} e^{i(2 k+1) \pi / p}$ and $z_{2}=t_{2} e^{i(2 k+1) \pi / p}$, where $t_{2}>t_{1}>0$ and $k \in$ $\{0,1, \cdots, p-1\}$, then

$$
\frac{\ln \left(\left|f^{n}\left(z_{2}\right)\right|+1\right)}{\ln \left(\left|f^{n}\left(z_{1}\right)\right|+2\right)} \rightarrow \infty \text { as } n \rightarrow \infty .
$$

It now follows from (1.1) that $z_{1}$ cannot belong to a Baker domain and, from hyperbolic metric estimates (see [16]), that $z_{1}$ cannot belong to any component of $N(f)$. This proves part (a).

To prove part (b), we use the fact that $(f(z))^{p}=g\left(z^{p}\right)$, where

$$
g(z)=a^{p} z\left(1+e^{-z}\right)^{p}=a^{p} z+p a^{p} z e^{-z}(1+o(1)),
$$

as $\Re(z) \rightarrow \infty$. By Theorem $2, g$ has a simply connected invariant Baker domain $U$ which, for each $\rho>0$ and large values of $R>0$, contains a set of the form

$$
D_{\rho, R}=\left\{z:\left|z e^{-z}\right|<\rho,|z|>R\right\}
$$

Also, since $g$ has no finite asymptotic values, there are infinitely many critical points of $g$ in $U$.

It follows that $f$ has a simply connected invariant Baker domain $U_{0}$ in $A_{0}$ which, for each $\epsilon, 0<\epsilon<\frac{\pi}{2 p}$, contains a set of the form

$$
E_{\epsilon, R}=\left\{z:|\arg z|<\frac{\pi}{2 p}-\epsilon,|z|>R\right\},
$$

and that there are infinitely many critical points of $f$ in $U_{0}$. Also, for $k=$ $0,1, \cdots, p-1$, each $U_{k}=e^{2 \pi k i / p} U_{0}$ is such an invariant Baker domain in $A_{k}$.

If $f$ has other invariant Baker domains, then by symmetry, part (a) and the fact that the positive real axis lies in $U$, there is one, $V$ say, which lies between $U_{0}$ and $\{z: \arg z=\pi / p\}$. Since $f$ is entire, $V$ is simply connected and so, by (1.1), $V$ contains a path $\Gamma$ tending to $\infty$ of the form

$$
\Gamma=\bigcup_{n=0}^{\infty} f^{n}\left(\Gamma_{0}\right)
$$

where $\Gamma_{0}$ joins $z_{0}$ to $z_{1}=f\left(z_{0}\right)$, such that, for some $C>0$,

$$
|f(z)| \leq C|z|, \text { for } z \in \Gamma \text {. }
$$

Now let

$$
B_{\epsilon}=\left\{z:\left|\arg z-\frac{\pi}{p}\right|<\frac{\pi}{2 p}-\epsilon\right\}, 0<\epsilon<\frac{\pi}{2 p} .
$$

For $z \in B_{\epsilon}$, we have

$$
\frac{\pi}{2}+p \epsilon<\arg \left(z^{p}\right)<\frac{3 \pi}{2}-p \epsilon
$$


so that, for such $z$,

$$
|f(z)| \geq a|z|\left(\exp \left(\Re\left(-z^{p}\right)\right)-1\right) \geq a|z|\left(\exp \left(\frac{2 \epsilon p}{\pi}|z|^{p}\right)-1\right) .
$$

From this, (4.1), the fact that $U_{0} \cap V=\emptyset$ and (4.2), we deduce that

$$
\arg z \rightarrow \frac{\pi}{2 p} \text { as } z \rightarrow \infty \text { along } \Gamma \text {. }
$$

Hence

$$
\arg \left(-z^{p}\right) \rightarrow-\frac{\pi}{2} \text { as } z \rightarrow \infty \text { along } \Gamma .
$$

Thus, if $h(z)=e^{-z^{p}}$, then $h(\Gamma)$ winds infinitely often round 0 . In particular, there is a sequence $\xi_{n} \in \Gamma$ tending to $\infty$ such that $e^{-\xi_{n}^{p}}$ is real and positive. Now

$$
\left|f\left(\xi_{n}\right)-\xi_{n}\right|=\left|a \xi_{n}\left(1+e^{-\xi_{n}^{p}}\right)-\xi_{n}\right| \geq(a-1)\left|\xi_{n}\right| .
$$

Thus, by Theorem 1 part (b),

$$
V \supset \bigcup_{n=0}^{\infty} B\left(\xi_{n}, c(a-1)\left|\xi_{n}\right|\right),
$$

for some $c>0$. Together with (4.3), this implies that, if $\epsilon>0$ is sufficiently small, then $V \cap E_{\epsilon, R} \neq \emptyset$ and hence $V \cap U_{0} \neq \emptyset$. This, however, is a contradiction, and so the proof of Theorem 3 is complete.

\section{Proof of Theorem 4}

Recall that Theorem 4 states that, for each $p \in \mathbf{N}$, there exists an entire function $f$ which has a $p$-cycle of Baker domains on which $f$ is univalent. To prove this result we consider, for $k \in\{0,1, \ldots, p-1\}$, the truncated sector

$$
S_{k}=\left\{z:|z| \geq \frac{3}{4},|\arg z-2 k \pi / p| \leq \frac{\pi}{2 p}\right\},
$$

and put $\omega_{k}=e^{2 \pi i k / p}$

$$
f_{k}(z)=a\left(z-\omega_{k}\right)+\omega_{k}, \quad g_{k}(z)=b\left(z-\omega_{k}\right)+\omega_{k}, \quad h_{k}(z)=c\left(z-\omega_{k}\right)+\omega_{k},
$$

where the constants $a, b$ and $c$ are chosen so that $1<c<b<a<3 / 2$ and the sets $f_{k}\left(S_{k}\right)$ are mutually disjoint. Then put

$$
\begin{gathered}
S=\mathbf{C} \backslash \bigcup_{k=0}^{p-1} g_{k}\left(S_{k}\right), \\
T_{k}=\left\{z:\left|\arg \left(z-2 \omega_{k}\right)-2 k \pi / p\right| \leq \frac{\pi}{2 p}\right\},
\end{gathered}
$$

and

$$
\epsilon=\min \left\{\operatorname{dist}\left(f_{0}\left(\partial S_{0}\right), g_{0}\left(\partial S_{0}\right)\right), \operatorname{dist}\left(f_{0}\left(\partial T_{0}\right), g_{0}\left(\partial T_{0}\right)\right), \operatorname{dist}\left(\partial S_{0}, h_{0}\left(\partial S_{0}\right)\right)\right\} .
$$

In particular, $0<\epsilon<a-1<1 / 2$.

It follows from Arakelyan's Theorem [12] that there exists an entire function $f$ such that

$$
\left|f(z)-f_{k}(z)\right|<\epsilon, \text { for } z \in h_{k}\left(S_{k}\right), k=0,1, \ldots, p-1
$$

and

$$
|f(z)|<\epsilon, \text { for } z \in S
$$


Since we may assume that $f$ is symmetric under rotation by $\omega_{1}$, we need only consider the case $k=0$ from now on. Condition (5.1) implies that $f\left(\partial S_{0}\right) \subset S$ and condition (5.2) then implies that $\partial S_{0}$ is contained in an attracting component of $N(f)$. On the other hand, condition (5.1) and the fact that $b>1$ implies that $f\left(T_{0}\right) \subset g_{0}\left(T_{0}\right) \subset T_{0}$ and, for $z \in T_{0}$,

$$
\begin{aligned}
\Re(f(z)-z) & \geq \Re\left(f_{0}(z)-z\right)-\left|f_{0}(z)-f(z)\right| \\
& \geq a-1-\epsilon>0 .
\end{aligned}
$$

Thus $T_{0}$ must be a subset of an invariant Baker domain $U_{0}$ for $f$, which is disjoint from $\partial S_{0}$.

We now check that $f$ is univalent on $S_{0}$ and hence on $U_{0}$. If we write $f(z)=$ $a(z-1)+1+\phi(z)$, then $|\phi(z)|<\epsilon$ on $h_{0}\left(S_{0}\right)$ and so $\left|\phi^{\prime}(z)\right|<\epsilon / \epsilon=1$ on $S_{0}$. Thus, if $z_{1}, z_{2} \in S_{0}$ with $f\left(z_{1}\right)=f\left(z_{2}\right)$, then

$$
a\left|z_{1}-z_{2}\right|=\left|\phi\left(z_{1}\right)-\phi\left(z_{2}\right)\right| \leq\left|z_{1}-z_{2}\right|
$$

and so $z_{1}=z_{2}$ as required.

We end by observing that the function $\omega_{1} f$ has the required properties.

\section{REFERENCES}

[1] I. N. Baker, Wandering domains in the iteration of entire functions. Proc. London Math. Soc. (3) 49 (1984), 563-576. MR 86d:58066

[2] I. N. Baker and J. Weinreich, Boundaries which arise in the iteration of entire functions. Rev. Roumaine Math. Pures Appl., 36 (1991), 413-420. MR 93e:30056

[3] A. F. Beardon, Iteration of Rational Functions, Springer, 1991. MR 92j:30026

[4] W. Bergweiler, Iteration of meromorphic functions, Bull. Amer. Math. Soc., 29 (1993), 151-188. MR 94c:30033

[5] W. Bergweiler, Invariant domains and singularities, Math. Proc. Camb. Phil. Soc., 117 (1995), 525-532. MR 96b:30055

[6] W. Bergweiler, On the Julia set of analytic self-maps of the punctured plane, Analysis, 15 (1995), 251-256. MR 96k:30028

[7] L. Carleson and T. W. Gamelin, Complex Dynamics, Springer, 1993. MR 94h:30033

[8] P. Dominguez, Dynamics of transcendental meromorphic functions, Ann. Acad. Sci. Fenn. Math., (1) 23 (1998), 225-250. MR 99b:30031

[9] P. Dominguez and I. N. Baker, Boundaries of unbounded Fatou components of entire functions, To appear in Ann. Acad. Sci. Fenn. Math.

[10] A. E. Erememko and M. Yu. Lyubich, Examples of entire functions with pathological dynamics, J. London Math. Soc., (2) 36 (1987), 458-468. MR 89e:30047

[11] P. Fatou, Sur l'itération des fonctions transcendantes entières, Acta. Math. France, 47 (1926), 337-360.

[12] D. Gaier, Lectures on Complex Approximation, Birkhäuser, 1985.

[13] M. Herman, Are there critical points on the boundary of singular domains? Comm. Math. Phys., 99 (1985), 593-612. MR 86j:58067

[14] C. Pommerenke, On the iteration of analytic functions in a halfplane, I, J. London Math. Soc. (2), 19 (1979), 439-447. MR 83j:30023

[15] P. J. Rippon and G. M. Stallard, Families of Baker domains, I. To appear in Nonlinearity.

[16] P. J. Rippon and G. M. Stallard, On sets where iterates of a meromorphic function zip towards infinity. Preprint.

Department of Pure Mathematics, The Open University, Walton Hall, Milton Keynes, MK7 6AA ENGLAND

E-mail address: p.j.rippon@open.ac.uk

E-mail address: g.m.stallard@open.ac.uk 